\title{
Impact of azelnidipine treatment on left ventricular diastolic performance in patients with hypertension and mild diastolic dysfunction: multi-center study with echocardiography
}

\author{
Hiroshi Ito ${ }^{1}$, Katsuhisa Ishii ${ }^{2}$, Katsuomi Iwakura ${ }^{3}$, Fumiaki Nakamura ${ }^{4}$, Toshihiko Nagano ${ }^{5}$ \\ and Shin Takiuchi ${ }^{6}$, for the Clinical impact of Azelnidipine on Left VentricuLar diastolic function \\ and OutComes in patients with hypertension (CALVLOC) trial investigators ${ }^{7}$
}

We investigated the impact of lowering blood pressure (BP) with azelnidipine, a newly developed calcium channel blocker, generation on the left ventricular (LV) diastolic function and LV filling pressure by assessing non-invasive indices derived from echo Doppler study. This study evaluated 232 hypertensive patients with diastolic dysfunction. This study had two groups: (1) in which azelnidipine was administered to patients as a first-line therapy, and (2) in which amlodipine was converted to azelnidipine. Early diastolic mitral annulus velocity $\left(e^{\prime}, \mathrm{cm} \mathrm{s}^{-1}\right)$, the ratio of peak $E$ velocity to $e^{\prime}$ velocity ( $E / e^{\prime}$ ratio) and level of brain natriuretic peptide (BNP) were measured before and, an average of, 8 months after azelnidipine treatment. In the first-line azelnidipine group, the systolic and diastolic BP reduced by 26 and $11 \mathrm{~mm} \mathrm{Hg}$, respectively. The $e^{\prime}$ increased, and $E / e^{\prime}$ ratio and BNP level decreased significantly. In the converted-from-amlodipine group, the systolic and diastolic BP decreased by 14 and $6 \mathrm{mmHg}$, respectively. The $e^{\prime}$ velocity increased, but the $E / e^{\prime}$ ratio and BNP level did not change. In both groups, azelnidipine lowered BP and improved LV diastolic function (an increase in the $e^{\prime}$ velocity). Possible reduction in LV filling pressure (a decrease in the $E / e^{\prime}$ ratio and BNP level) is observed only in the first-line azelnidipine group. Hypertension Research (2009) 32, 895-900; doi:10.1038/hr.2009.119; published online 14 August 2009

Keywords: calcium channel blocker; diastolic function; echocardiography; heart failure

\section{INTRODUCTION}

Left ventricular diastolic dysfunction is possibly the most common form of heart failure. ${ }^{1,2}$ Patients with hypertension are at an increased risk of developing left ventricular hypertrophy, myocardial fibrosis and chronic heart failure due to diastolic dysfunction. Up to $50 \%$ of patients with hypertension have evidence of diastolic dysfunction. ${ }^{3}$ The treatment of diastolic dysfunction using antihypertensive drugs represents an attractive method for the prevention of heart failure. To date, no specific treatments have been definitively shown to improve the diastolic function or to reduce mortality and heart failure event. ${ }^{4}$ The increase in the prevalence of heart failure with preserved systolic function over time and the stability in the incidence of death or heart failure from this condition underline the importance of studies to develop therapeutic strategies against it. The long-term treatment of hypertension results in a regression of existing left ventricular hypertrophy and, therefore, the role of the inhibition of the renin-angiotensin-aldosterone system is addressed in relation to the treatment of diastolic dysfunction. ${ }^{5,6}$ In the Valsartan in Diastolic Dysfunction (VALIDD) trial, however, the inhibition of the renin-angiotensin system failed to show an additional benefit after the change in diastolic relaxation velocity in comparison with the antihypertensive drugs of non-rennin-angiotensin system inhibition. ${ }^{7}$ This study showed that lowering blood pressure (BP) improves the diastolic function irrespective of the type of antihypertensive agent used.

Calcium channel blockers are common antihypertensive drugs. We recognized that, in general, calcium channel blocker does not have a favorable effect on heart failure due to diastolic dysfunction. A newly developed calcium channel blocker, azelnidipine, is very long acting, and has no or little influence on either the sympathetic nerve system or the renin-angiotensin system. This is explained by its unique nature referred to as the membrane approach. ${ }^{8}$ The aim of this trial was to

${ }^{1}$ Department of Cardiovascular Medicine, Okayama University, Graduate School of Medicine, Okayama, Japan; ${ }^{2}$ Division of Cardiology, Kansai Electric Power Hospital, Osaka, Japan; ${ }^{3}$ Cardiovascular Center, Sakurabashi Watanabe Hospital, Osaka, Japan; ${ }^{4}$ Division of Medicine, Ibaraki Iseikai Hospital, Ibaraki, Japan; ${ }^{5}$ Internal Medicine, Iwasa Dai-ichi Hospital, Gifu, Japan and ${ }^{6}$ Division of Cardiology, Higashi-Takarazuka Sato Hospital, Takarazuka, Japan

${ }^{7}$ CALVLOC trial investigators list is provided in the Appendix.

Correspondence: Professor H Ito, Department of Cardiovascular Medicine, Okayama University, Graduate School of Medicine, 2-5-1 Kita-ku, Shikata-cho, Okayama 700-8558, Japan. E-mail: itomd@md.okayama-u.ac.jp

Received 20 April 2009; revised 15 June 2009; accepted 21 June 2009; published online 14 August 2009 
investigate the impact of lowering $\mathrm{BP}$, using azelnidipine, on the diastolic function and left ventricular filling pressure in patients with preserved ejection fraction and diastolic dysfunction by assessing non-invasive indices derived from echo Doppler study. The influence of azelnidipine on the diastolic function was also compared with that of amlodipine., 9,10

\section{METHODS}

\section{Study population}

Men and women, aged 20-80 years, with a history of stage 1 or 2 essential hypertension (mean BP measurement of $>140 \mathrm{~mm} \mathrm{Hg}$ systolic or $>90 \mathrm{~mm} \mathrm{Hg}$ diastolic) were screened for inclusion by assessing their systolic and diastolic function by echocardiography. Echocardiographic assessment of mitral annular relaxation velocity was used to make a diagnosis of diastolic dysfunction. The exclusion criteria for exclusion were left ventricular ejection fraction of $<50 \%$, septal mitral annular relaxation velocity $>8 \mathrm{~cm} \mathrm{~s}^{-1}$, atrial fibrillation and the administration of calcium channel blockers other than amlodipine. The study protocol was approved by individual sites and written informed consent was obtained from each patient before the study began.

\section{Study protocol}

There were two groups in this study. In patients not on antihypertensive drugs or on antihypertensive drugs, but not calcium channel blocker, $16 \mathrm{mg}$ of azelnidipine, which is the routine clinical dose, was administered. In patients treated with amlodipine, amlodipine was stopped and substituted with $16 \mathrm{mg}$ of azelnidipine. No other medications were changed throughout the study period. Patients were assessed at 4-8 week intervals for, at least, 24 weeks, and underwent echocardiographic assessment at the end of the study. All patients underwent echocardiographic screening for systolic and diastolic function before the start of azelnidipine treatment. The diastolic function was assessed by tissue Doppler imaging of septal mitral annular velocity during diastole. Blood and urine tests were done at baseline and at the end of the study.

BP and heart rate were measured in a sitting position at each study visit. The adequacy of antihypertensive therapy was determined on the basis of measured BP. BP was also measured at the time of echocardiographic assessment. The use of concomitant antihypertensive medication was recorded at each study visit. If the $\mathrm{BP}$ was not adequately controlled, then add-on treatment was considered, but such patients dropped out from this study.

\section{Echo data analysis}

All patients underwent the Doppler echocardiographic examination including tissue Doppler imaging. Doppler echocardiographic assessment included the peak velocities of E and A wave on mitral pulsed wave Doppler. Spectral pulsed wave Doppler tissue interrogation of longitudinal mitral annular velocity was recorded throughout the cardiac cycle at the septal annulus in the apical four-chamber view. Additional exploratory analyses included changes in the chamber dimensions and left ventricular ejection fraction, the ratio of peak mitral Doppler inflow velocities (E/A ratio) and the deceleration time of the $\mathrm{E}$ wave. Left ventricular mass was estimated from left ventricular dimension and was calculated as an index of body surface area in $\mathrm{m}^{2}$. The relative wall thickness was calculated as $2 \times$ (posterior wall thickness in diastole)/ (left ventricular end-diastolic diameter). ${ }^{11}$

The primary end point was a change in septal mitral annular velocity during diastole, the $e^{\prime}$ velocity and the ratio of mitral inflow velocity to the $e^{\prime}$ velocity $\left(E / e^{\prime} \text { ratio }\right)^{12}$ from the baseline to the follow-up. Secondary efficacy measures included differences in changes in blood pressure, heart rate, wall thickness, left ventricular mass index and left atrial volume index from the baseline to the follow-up. Blood samples were collected for assessment of brain natriuretic peptide (BNP) and high sensitivity C-reactive protein along with additional exploratory blood analyses, and urine was collected for assessing the albumin concentration.

\section{Statistical analysis}

All of the results are expressed as mean \pm s.d. and proportions (\%). Results are expressed as median (interquartile range) when studying the relationship of changes in the $e^{\prime}$ velocity and $E / e^{\prime}$ ratio with the reduction in systolic blood pressure or left ventricular mass index. Student's $t$-test was used for parametric data when normal distribution and equal dispersion were recognized. Welch's $t$-test was used when the variance was unequal. Plasma BNP level was also expressed as median (interquartile range) and was analyzed non-parametrically (Wilcoxon's test). Differences in the categorical data were analyzed by $\chi^{2}$-analyses, and Fisher's exact test was used when appropriate. Differences were considered to be statistically significant when the $P$-values were $<0.05$.

\section{RESULTS}

After echocardiographic screening, 253 patients with a history of treated or untreated hypertension were enrolled in this study between January 2006 and October 2007. Twenty-one patients were excluded from this study, as 15 patients were lost to follow-up and 6 patients had to be removed for protocol violation. Thus, the follow-up study was completed in 232 (92\%) patients. LV concentric remodeling (relative wall thickness $\geqslant 0.47 \mathrm{~cm}$ ) was present in $97(41 \%)$ patients. The baseline characteristics, medication use and echocardiographic parameters are shown in Tables 1 and 2. Mild heart failure (New York Heart Association class 2 (NYHA-2)) was found in 29 patients and no patients showed moderate to severe heart failure. Average interval between baseline and follow-up was 8 months (range 6-10 months). No patient died or developed clinical heart failure, had myocardial infarction or developed hypotension during the course of the study. In addition, no patient showed any investigator-reported serious adverse events.

Table 1 Baseline clinical and demographic characteristics of the patients

\begin{tabular}{lc}
\hline Continuous & \\
Age (years) & $63 \pm 32$ \\
Height $(\mathrm{cm})$ & $158.5 \pm 24.5$ \\
Weight $(\mathrm{kg})$ & $69.5 \pm 36.5$ \\
Waist length $(\mathrm{cm})$ & $91.5 \pm 33.5$ \\
Body mass index $\left(\mathrm{kg} \mathrm{m}^{-1}\right)$ & $24.5+3.3$ \\
Abdominal circumference (cm) & $91.5 \pm 33.5$ \\
Systolic BP (mm Hg) & $160 \pm 17$ \\
Diastolic BP (mm Hg) & $87 \pm 14$ \\
Heart rate (beats per $\mathrm{min})$ & $73 \pm 10$
\end{tabular}

Demographics categorical

Gender (\% male)

Definite diabetes (\%)

Hypercholesteremia (\%)

Current smoking (\%)

Angina pectoris (\%)

History of myocardial infarction (\%)

History of cerebral infarction (\%)

NYHA class 2

$\begin{array}{lc}\text { Antihypertensive drugs used } & \\ \text { RAS inhibitors (\%) } & 52 \\ \beta \text {-Blocker (\%) } & 15 \\ \text { Thiazide (\%) } & 7 \\ \text { Statin (\%) } & 32\end{array}$

Number of antihypertensive drugs

2 or more 
Table 2 Echocardiographic characteristics at the baseline examination

\begin{tabular}{lc}
\hline LVDd $(\mathrm{cm})$ & $46.6 \pm 5$ \\
LVDs $(\mathrm{cm})$ & $28.4 \pm 4.4$ \\
Septal thickness $(\mathrm{cm})$ & $10.0 \pm 1.6$ \\
Posterior wall thickness $(\mathrm{cm})$ & $9.9 \pm 1.7$ \\
RWT & $0.43 \pm 0.07$ \\
Left ventricular mass index $\left(\mathrm{g} \mathrm{m}^{-2}\right)$ & $94.7 \pm 36.7$ \\
Left atrial dimension $(\mathrm{cm})$ & $35.7 \pm 5.5$ \\
Left atrial volume index $\left(\mathrm{ml} \mathrm{m}^{-2}\right)$ & $21.5 \pm 9.3$ \\
Peak $E\left(\mathrm{~cm} \mathrm{~s}^{-1}\right)$ & $65 \pm 16$ \\
Peak $A,\left(\mathrm{~cm} \mathrm{~s}^{-1}\right)$ & $82 \pm 16$ \\
E/A ratio & $0.83 \pm 0.24$ \\
Deceleration time $(\mathrm{ms})$ & $230 \pm 56$ \\
$E^{\prime}$ velocity $\left(\mathrm{cm} \mathrm{s}^{-1}\right)$ & $6.0 \pm 1.4$ \\
Ele' ratio & $11.5 \pm 3.8$ \\
\hline
\end{tabular}

Abbreviations: LVDd, left ventricular dimension at end diastole; LVD, left ventricular dimension at end systole; RWT, relative wall thickness.

Data are presented as the mean \pm s.d.

\section{Analysis of the first-line azelnidipine group}

This group consisted of 160 patients. At follow-up, the systolic and diastolic blood pressures had decreased from baseline by 26 and $11 \mathrm{~mm} \mathrm{Hg}$, respectively. Heart rate decreased by 3 beats per minute. There were no significant changes in left ventricular wall thickness, left ventricular mass index, ejection fraction or left atrial volume from baseline to follow-up. In addition, the peak $E$, peak $A$, deceleration time and $E / A$ ratio showed no significant change at follow up. From baseline to follow-up, the $e^{\prime}$ velocity significantly increased $(6.1 \pm 1.3$ vs. $6.8 \pm 1.2 \mathrm{~cm} / \mathrm{s}, P<0.0001)$, and the $E / e^{\prime}$ ratio and $\mathrm{BNP}$ decreased significantly $\left(E / e^{\prime}\right.$ ratio: $11.6 \pm 3.9$ vs. $10.1 \pm 3.0, P=0.0002$; BNP: 20.6 (12.5-36.3) vs. 14.9 (10.1-26.2) pg ml $\left.{ }^{-1}, P=0.0040\right)$. There were no significant changes, from baseline to follow-up, in the serum creatinine level, urine albumin concentration and high-sensitivity C-reactive protein level. See Table 3 for details.

\section{Analysis of the converted-from-amlodipine-to-azelnidipine group}

This group consisted of 72 patients. At follow-up, the systolic and diastolic blood pressures were observed to have decreased by 14 and $6 \mathrm{~mm} \mathrm{Hg}$, respectively, and heart rate decreased by 6 beats per minute. From baseline to follow-up, the $e^{\prime}$ velocity was found to have increased significantly $\left(6.1 \pm 1.3\right.$ vs. $\left.6.7 \pm 1.3 \mathrm{~cm} \mathrm{~s}^{-1}, P=0.011\right)$. The $E / e^{\prime}$ ratio decreased, but the difference did not reach statistical difference. There were no changes in the other echo Doppler parameters at the follow-up. There were no significant changes from baseline to follow-up in the BNP level (20.5 (11.2-35.8) vs. 15.7 (9.2-40.5) pg/ml, $P=0.28)$, serum creatinine level, urine albumin concentration and high-sensitivity C-reactive protein level. See Table 4, for details.

\section{Factors related to changes in $e^{\prime}$ velocity and $E / e^{\prime}$ ratio}

The impact of the reduction in blood pressure on the changes in the $e^{\prime}$ velocity and the E/e $e^{\prime}$ ratio was studied (Figure 1). A reduction in systolic blood pressure was associated with increases in the $e^{\prime}$ velocity in all study patients (q1 vs. q2 vs. q3 vs. q4 (median (interquartile range); $0.90(0.17-2.04)$ vs. $0.44(0.05-1.32)$ vs. 0.29 (0.05-1.12) vs. 0.10 ( -0.25 to 0.84$) \mathrm{cm} \mathrm{s}^{-1}, P=0.001$ ). In addition, a reduction in the systolic blood pressure was also associated with a decrease in the E/e' ratio $(-1.50(-3.05$ to -0.18$)$ vs. $-0.60(-3.00$ to -0.00$)$ vs. -0.29 ( -2.77 to 0.09 ) vs. 0.00 ( -1.95 to 1.69$), P=0.03$ ).

We analyzed the factors associated with the increase in the $e^{\prime}$ velocity. Factors included age, entry criteria, sex, risk factors, systolic blood
Table 3 Temporal changes in the hemodynamic, laboratory and echocardiographic parameters: first-line azelnidipine group

\begin{tabular}{lccr}
\hline & Pre & Post & P \\
\hline Systolic BP (mm Hg) & $164 \pm 15$ & $138 \pm 11$ & $<0.0001$ \\
Diastolic BP (mm Hg) & $89 \pm 13$ & $78 \pm 10$ & $<0.0001$ \\
Heart rate (b.p.m.) & $73 \pm 10$ & $70 \pm 9$ & 0.0054 \\
BNP (pg ml-1) & $37.8 \pm 61.8$ & $26.0 \pm 32.8$ & 0.0360 \\
Crn (mg 100 ml-1) & $0.84 \pm 0.34$ & $0.86 \pm 0.34$ & 0.6438 \\
HsCRP & $0.14 \pm 0.19$ & $0.11 \pm 0.11$ & 0.0513 \\
Urine albumine & $56 \pm 226$ & $51 \pm 229$ & 0.8629 \\
& & & \\
Echo Doppler parameters & & & \\
LVDd (cm) & $46.8 \pm 4.5$ & $46.1 \pm 4.1$ & 0.1538 \\
LVDs (cm) & $28.6 \pm 4.7$ & $28.0 \pm 3.7$ & 0.1930 \\
Septal thickness (cm) & $10.1 \pm 1.7$ & $9.6 \pm 1.6$ & 0.0184 \\
Posterior wall thickness (cm) & $9.9 \pm 1.8$ & $9.6 \pm 1.5$ & 0.1451 \\
RWT & $0.43 \pm 0.06$ & $0.43 \pm \pm 0.06$ & 0.8556 \\
LV mass index, (gcm ${ }^{-2}$ ) & $93 \pm 38$ & $92 \pm 40$ & 0.5879 \\
Left atrial dimension (cm) & $35.7 \pm 5.6$ & $35.2 \pm 4.9$ & 0.3851 \\
Left atrial volume index (ml m-2) & $21.6 \pm 9.6$ & $20.6 \pm 8.2$ & 0.3354 \\
Peak $E$ (cm s ${ }^{-1}$ ) & $66 \pm 16$ & $67 \pm 15$ & 0.7432 \\
Peak $A$ (cm s ${ }^{-1}$ ) & $81 \pm 16$ & $78 \pm 16$ & 0.0567 \\
E/A ratio & $0.85 \pm 0.25$ & $0.89 \pm 0.24$ & 0.1182 \\
Deceleration time (ms) & $229 \pm 58$ & $219 \pm 50$ & 0.1168 \\
$E^{\prime}$ velocity, (cm s ${ }^{-1}$ ) & $6.0 \pm 1.4$ & $6.8 \pm 1.3$ & $<0.0001$ \\
Ele' ratio & $11.6 \pm 3.9$ & $10.1 \pm 3.0$ & 0.0002 \\
\hline
\end{tabular}

Abbreviations: BNP, brain natriuretic peptide; b.p.m., beats per minute; hs-CRP, high-sensitive $C$ reactive protein; LVDd, left ventricular dimension at end diastole; LVD, left ventricular dimension at end systole; RWT, relative wall thickness.

Data are presented as the mean \pm s.d.

Table 4 Temporal changes in the physical and echo-Doppler parameters in patients in whom amlodipine was changed to azelnidipine

\begin{tabular}{|c|c|c|c|}
\hline & Pre & Post & $\mathrm{P}$ \\
\hline Systolic BP (mm Hg) & $153 \pm 16$ & $139 \pm 11$ & $<0.0001$ \\
\hline Diastolic BP (mm Hg) & $84 \pm 14$ & $78 \pm 9$ & $<0.0001$ \\
\hline Heart rate (b.p.m.) & $75 \pm 9$ & $69 \pm 10$ & 0.0005 \\
\hline $\mathrm{BNP}\left(\mathrm{pg} \mathrm{ml^{-1 }}\right)$ & $38 \pm 65$ & $34 \pm 47$ & 0.792 \\
\hline Crn (mg $100 \mathrm{ml}^{-1}$ ) & $1.014 \pm 0.94$ & $0.99 \pm 0.92$ & 0.8963 \\
\hline hsCRP & $0.16 \pm 0.19$ & $0.15 \pm 0.22$ & 0.8545 \\
\hline Urine albumin & $54 \pm 113$ & $39 \pm 78$ & 0.3507 \\
\hline \multicolumn{4}{|l|}{ Echo Doppler parameters } \\
\hline LVDd (cm) & $46.2 \pm 4.8$ & $46.3 \pm 4.4$ & 0.8900 \\
\hline LVDs $(\mathrm{cm})$ & $28.0 \pm 3.8$ & $27.7 \pm 4.7$ & 0.6514 \\
\hline Septal thickness $(\mathrm{cm})$ & $9.9 \pm 1.5$ & $9.9 \pm 1.7$ & 0.8972 \\
\hline Posterior wall thickness $(\mathrm{cm})$ & $9.8 \pm 1.5$ & $9.9 \pm 1.5$ & 0.5273 \\
\hline RWT & $0.43 \pm 0.06$ & $0.43 \pm 0.06$ & 0.6597 \\
\hline LV mass index $\left(\mathrm{g} \mathrm{m}^{-2}\right)$ & $95 \pm 34$ & $96 \pm 37$ & 0.8796 \\
\hline Left atrial dimension $(\mathrm{cm})$ & $35.8 \pm 5.2$ & $35.7 \pm 5.3$ & 0.9208 \\
\hline Left atrial volume index $\left(\mathrm{ml} \mathrm{m}^{-2}\right)$ & $21.5 \pm 8.9$ & $21.4 \pm 9.8$ & 0.9397 \\
\hline Peak $E\left(\mathrm{~cm} \mathrm{~s}^{-1}\right)$ & $66 \pm 17$ & $67 \pm 18$ & 0.5590 \\
\hline Peak $A,\left(\mathrm{~cm} \mathrm{~s}^{-1}\right)$ & $85 \pm 18$ & $80 \pm 22$ & 0.1602 \\
\hline E/A ratio & $0.80 \pm 0.21$ & $0.86 \pm 0.22$ & 0.1697 \\
\hline Deceleration time (ms) & $233 \pm 49$ & $224 \pm 43$ & 0.2296 \\
\hline$E^{\prime}$ velocity, $\left(\mathrm{cm} \mathrm{s}^{-1}\right)$ & $6.1 \pm 1.3$ & $6.7 \pm 1.3$ & 0.0111 \\
\hline Ele' ratio & $11.2 \pm 3.4$ & $10.5 \pm 2.8$ & 0.1624 \\
\hline
\end{tabular}

Abbreviations: BNP, brain natriuretic peptide; b.p.m., beats per minute; hs-CRP, high-sensitive $C$ reactive protein; LVDd, left ventricular dimension at end-diastole; LVDs, left ventricular C reactive protein; LVDd, left ventricular dimension at
dimension at end-systole; RWT, relative wall thickness.

Data are presented as the mean \pm s.d. 

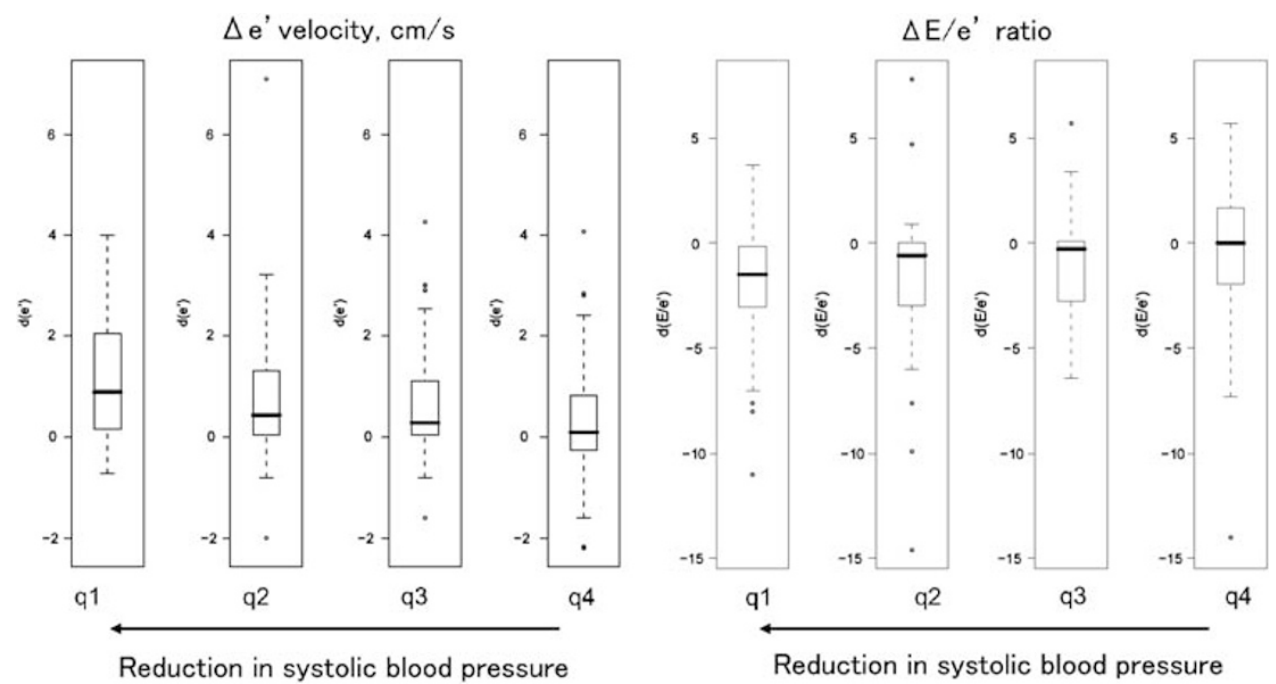

Figure 1 Impact of lowering systolic blood pressure on changes in the e' velocity and the E/e' ratio. The reduction in the systolic blood pressure was associated with increases in the $e^{\prime}$ velocity (left) and with the decrease in the E/e' ratio. Data are expressed as median and interquartile range.
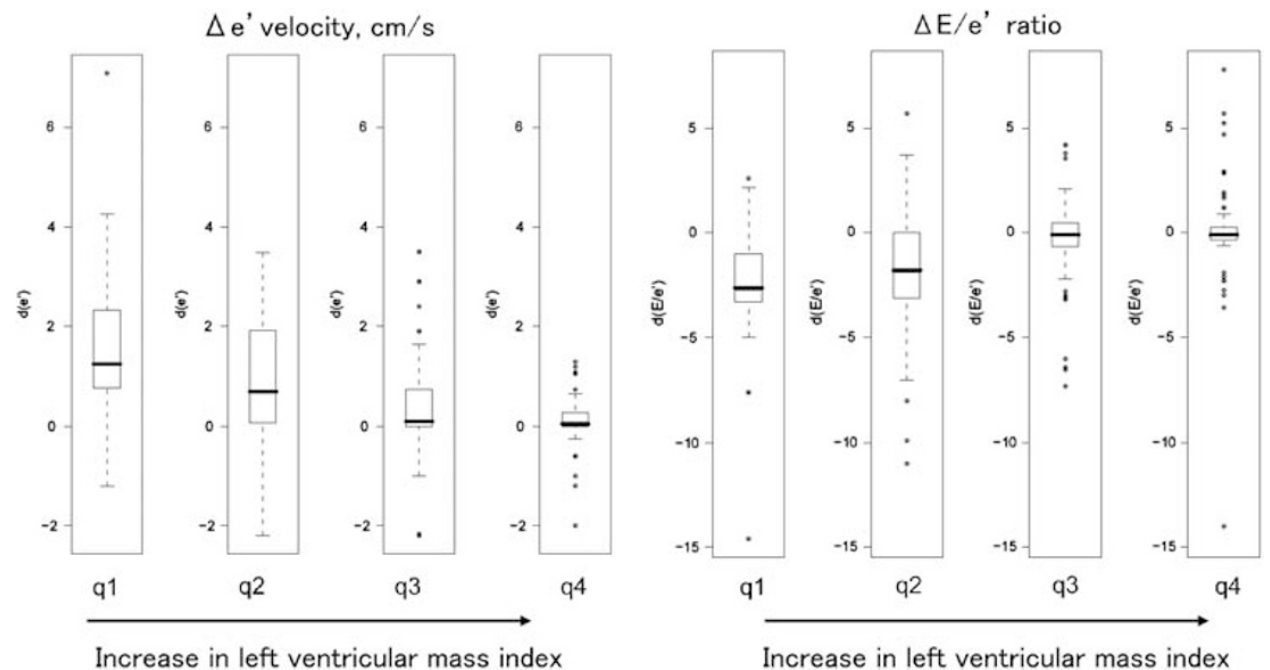

Figure 2 Impact of increasing the left ventricular mass index on changes in the $e^{\prime}$ velocity and the E/e' ratio. An increase in the left ventricular mass index was associated with lesser increase in the $e^{\prime}$ velocity (left) and with a smaller decrease in the E/e' ratio. Data are expressed as median and interquartile range.

pressure, high-sensitivity C-reactive protein level, left atrial volume, ventricular septal thickness, left ventricular mass and peak $E$ velocity. A multivariate analysis showed that the left ventricular mass index $(P=0.0001)$, angina pectoris $(P=0.015)$, ventricular septal thickness $(P=0.016)$, left atrial volume index $(P=0.030)$ and first-line azelnidipine $(P=0.043)$ were independent factors. The impact of left ventricular mass index on the change in the $e^{\prime}$ velocity was investigated. A greater left ventricular mass index was associated with a smaller increase in the $e^{\prime}$ velocity during azelnidipine treatment (Figure 2; $1.26(0.77-2.32)$ vs. $0.70(0.08-1.91)$ vs. $0.10(0.00-0.75)$ vs. $\left.0.05(0.00-0.28) \mathrm{cm} \mathrm{s}^{-1}, P<0.0001\right)$. We also analyzed the factors associated with the magnitude of increase in the $E / e^{\prime}$ ratio. A multivariate analysis showed that peak $E$ velocity $(P=0.0002)$, left ventricular mass index $(P=0.0006)$ and ventricular septal thickness $(P=0.043)$ were independent factors. The greater left ventricular mass index was associated with a smaller decrease in the $E / e^{\prime}$ ratio during azelnidipine treatment (Figure $2 ;-2.65(-3.30$ to -1.00$)$ vs. -1.80
( -3.13 to -0.03$)$ vs. -0.09 ( -0.67 to 0.47$)$ vs. $-0.10(-0.35$ to 0.26$)$, $P<0.0001)$.

\section{DISCUSSION}

We showed that lowering blood pressure with the additional use of azelnidipine is associated with an improvement in the left ventricular diastolic performance (an increase in the $e^{\prime}$ velocity), a reduction in left ventricular filling pressure (a decrease in the E/e ratio) and reduction in BNP level in patients with hypertension and with a preserved systolic function. Among the patients in whom amlodipine was converted to azelnidipine, blood pressure and heart rate significantly reduced and these reductions was associated with an increase in the $e^{\prime}$ velocity. This is the first clinical trial that shows a potential mechanism by which treating hypertension with azelnidipine might be useful to treat hypertensive patients with diastolic dysfunction.

The tissue Doppler assessment of myocardial relaxation was used as a primary end point in this study. The $e^{\prime}$ velocity is robust, 
non-invasive measure of the early diastolic relaxation during the early adenosine triphosphate-dependent active phases of diastole, and is less sensitive to preload than mitral flow profiles under physiological condition. The azelnidipine treatment was associated with an increase in the $e^{\prime}$ velocity both study arms. There are several mechanisms to explain the improvement in left ventricular diastolic function associated with azelnidipine treatment. First, the reduction in the systolic blood pressure, in itself, may be associated with the improvement in left ventricular diastolic function as shown in VALIDD trial. ${ }^{4,7,13-16}$ In this study, the greater reduction in systolic blood pressure was associated with the greater increase in the $e^{\prime}$ velocity. Second, left ventricular systolic load may be reduced by attenuating the wave reflection. An increase in the aortic stiffness because of hypertension is associated with the early return of wave reflection to exaggerate the second systolic peak and to increase systolic blood pressure. Elevated systolic load impairs left ventricular diastolic function and raises left ventricular filling pressure. Reducing aortic stiffness, using verapamil, reduced and delayed wave reflection and reduced the late systolic blood pressure. ${ }^{17,18}$ Third, azelnidipine may improve the vascular function by scavenging free radicals, by suppressing the oxidative stress and release of interleukin- 8 to reduce oxygen radical stress, and by enhancing the production of endothelial nitric oxide. ${ }^{19}$ Finally, long-acting calcium channel blockers may directly prevent the progression of the diastolic dysfunction. In an experimental study using Dahl salt-sensitive rats, long-term administration of amlodipine prevented the transition of diastolic heart failure possibly due to an amelioration of collagen remodeling. ${ }^{20}$

Factors that influence changes in the $e^{\prime}$ velocity and the E/e ratio with antihypertensive treatment remain unknown. In this study, we found that the left ventricular hypertrophy is a factor that attenuates the beneficial impact of azelnidipine on the diastolic function and left ventricular filling pressure. Our data implicated that greater left ventricular mass index was associated with the smaller increase in the $e^{\prime}$ velocity and with the smaller decrease in the $E / e^{\prime}$ ratio during azelnidipine treatment. In the highest left ventricular mass index quartile, the increase in the $e^{\prime}$ velocity was minimal and the $E / e^{\prime}$ ratio did not decrease. Therefore, the improvement in left ventricular diastolic function with azelnidipine treatment is expected especially in patients with either no or mild left ventricular hypertrophy. Echocardiographic assessment of left ventricular hypertrophy is useful to predict the responder of azelnidipine treatment.

In VALIDD trial, the E/e' ratio did not show significant change in both valsaltan group and non-RAS inhibitor treatment group, although the $e^{\prime}$ velocity increased in both groups. We studied the impact of azelnidipine treatment on the E/e $e^{\prime}$ ratio. ${ }^{7}$ As there is an overlap of the $E / e^{\prime}$ ratio of a level similar to that of left ventricular filling pressure, ${ }^{12}$ we focused on the temporal changes in $E / e^{\prime}$ ratio in each patient in this study. In the converted-from-amlodipine group, the $E / e^{\prime}$ ratio did not show significant change at follow-up, and BNP level also did not change. In the first-line azelnidipine group, the $E / e^{\prime}$ ratio significantly decreased, and this was associated with a reduction in BNP level, although the baseline BNP level was low. These data imply that azelnidipine does not worsen heart failure state and can be safely used in hypertensive patients with diastolic dysfunction.

\section{Limitations}

Some limitations should be noted. This study was not a double blind randomized trial. The main objective of this study was not to monitor the clinical outcomes but to assess the temporal changes in objective parameters to assess the left ventricular diastolic function associated with azelnidipine treatment. We could not conclude that azelnidipine has specific impact on the left ventricular diastolic function that is not observed in other calcium channel blockers. The possibility that similar findings would have been seen with short-term blood pressure lowering cannot be excluded, because no early measure was obtained. Most of the study patients were, mildly to moderately, hypertensive and without decompensated heart failure. These findings might not be applied to the severe diastolic heart failure population. In this study, the magnitude of increase in the $e^{\prime}$ velocity was less in those with the greater left ventricular hypertrophy. Whether an increase in mitral annulus velocity by $0.6-0.8 \mathrm{~cm} \mathrm{~s}^{-1}$ is clinically meaningful or, over time, would be associated with improvements in outcomes is not known. The dosage of azelnidipine might have influenced the results. The routine clinical dose of azelnidipine was used, and this dose achieved a similar reduction of blood pressure as the routine dose $(5 \mathrm{mg})$ of amlodipine. Finally, as the echo Doppler parameters used in this study are those that are routinely used, we did not assess the reproducibility of these parameters among centers.

\section{Clinical implications}

At present, renin-angiotensin system inhibitors are the first-line treatment for patients with hypertension with diastolic dysfunction. ${ }^{21,22}$ The inhibition of the renin-angiotensin system offers other benefits besides lowering blood pressure in a variety of disorders, such as myocardial fibrosis and hypertrophy. ${ }^{23,24}$ However, it is often difficult to manage blood pressure with renin-angiotensin system inhibitor alone, and we should use calcium channel blocker in some cases. In this situation, we should choose long-acting calcium channel blockers. In the Antihypertensive and Lipid-Lowering Treatment to Prevent Heart Attack Trial (ALLHAT), the rate of new-onset heart failure with preserved ejection fraction was comparable between amlodipine and lisinopril. ${ }^{25}$ Our study implied that lowering blood pressure with azelnidipine, in addition to renin-angiotensin system inhibitors, similar to other long-acting calcium channel blockers, could be an option for managing diastolic heart failure. We also showed that the hear rate reduced when amlodipine was converted to azelnidipine. The clinical implications of the decrease in heart rate using azelnidipine treatment require further investigations.

\section{ACKNOWLEDGEMENTS}

This study was supported by Japan Vascular Disease Research Foundation.

1 Ho KK, Pinsky JL, Kannel WB, Levy D. The epidemiology of heart failure: the Framingham Study. J Am Coll Cardiol 1993; 22(Suppl A): 6A-13A.

2 Levy D, Larson MG, Vasan RS, Kannel WB, Ho KK. The progression from hypertension to congestive heart failure. JAMA 1996; 275: 1557-1562.

3 Redfield MM, Jacobsen SJ, Burnett Jr JC, Mahoney DW, Bailey KR, Rodeheffer RJ. Burden of systolic and diastolic ventricular dysfunction in the community: appreciating the scope of the heart failure epidemic. JAMA 2003; 289: 194-202.

4 Aurigemma GP. Diastolic heart failure-a common and lethal condition by any name. N Engl J Med 2006; 20: 355:308-355:310.

5 Devereux RB, Dahlöf B, Gerdts E, Boman K, Nieminen MS, Papademetriou V, Rokkedal J, Harris KE, Edelman JM, Wachtell K. Regression of hypertensive left ventricular hypertrophy by losartan compared with atenolol: the Losartan Intervention for Endpoint Reduction in Hypertension (LIFE) trial. Circulation 2004; 110: 1456-1462.

6 Dahlof B, Pennert K, Hansson L. Reversal of left ventricular hypertrophy in hypertensive patients. A metaanalysis of 109 treatment studies. Am J Hypertens 1992; 5: 95-110.

7 Solomon SD, Janardhanan R, Verma A, Bourgoun M, Daley WL, Purkayastha D, Lacourcière Y, Hippler SE, Fields H, Naqvi TZ, Mulvagh SL, Arnold JM, Thomas JD, Zile MR, Aurigemma GP. Effect of angiotensin receptor blockade and antihypertensive drugs on diastolic function in patients with hypertension and diastolic dysfunction: a randomised trial. Lancet 2007; 369: 2079-2087.

8 Arita M, Hashizume T, Tanigawa K, Yamamoto H, Nishio I. A new Ca-antagonist, azelnidipine, reduced blood pressure during exercise without augmentation of sympathetic nervous system in essential hypertension: a randomized, double-blind, placebo-controlled trial. J Cardiovasc Pharmacol 1999; 33: 186-192. 
9 Sohn DW, Chai IH, Lee DJ, Kim HC, Kim HS, Oh BH, Lee MM, Park YB, Choi YS, Seo JD, Lee YW. Assessment of mitral annulus velocity by Doppler tissue imaging in the evaluation of left ventricular diastolic function. J Am Coll Cardiol 1997; 30: 474-480.

10 Garcia MJ, Thomas JD, Klein AL. New Doppler echocardiographic applications for the study of diastolic function. J Am Coll Cardiol 1998; 32: 865-875.

11 Lang RM, Bierig M, Devereux RB, Flachskampf FA, Foster E, Pellikka PA, Picard MH, Roman MJ, Seward J, Shanewise JS, Solomon SD, Spencer KT, Sutton MS, Stewart WJ. Recommendations for chamber quantification: a report from the American Society of Echocardiography's Guidelines and Standards Committee and the Chamber Quantification Writing Group, developed in conjunction with the European Association of Echocardiography, a branch of the European Society of Cardiology. J Am Soc Echocardiogr 2005; 18: 1440-1463.

12 Nagueh SF, Middleton KJ, Kopelen HA, Zoghbi WA, Quinones MA. Doppler tissue imaging: a noninvasive technique for evaluation of left ventricular relaxation and estimation of filling pressures. J Am Coll Cardiol 1997; 30: 1527-1533.

13 Setaro JF, Zaret BL, Schulman DS, Black HR, Soufer R. Usefulness of verapamil for congestive heart failure associated with abnormal left ventricular diastolic filling and normal left ventricular systolic performance. Am J Cardiol 1990; 66: 981-986.

14 Onose Y, Oki T, Yamada H, Manabe K, Kageji Y, Matsuoka M, Yamamoto T, Tabata T, Wakatsuki T, Ito S. Effect of cilnidipine on left ventricular diastolic function in hypertensive patients as assessed by pulsed Doppler echocardiography and pulsed tissue Doppler imaging. Jpn Circ J 2001; 65: 305-309.

15 Aronow WS, Ahn C, Kronzon I. Effect of propranolol versus no propranolol on total mortality plus nonfatal myocardial infarction in older patients with prior myocardial infarction, congestive heart failure, and left ventricular ejection fraction $>$ or $=40 \%$ treated with diuretics plus angiotensin-converting enzyme inhibitors. Am J Cardiol 1997; 80: 207-209.

16 Friedrich SP, Lorell BH, Rousseau MF, Hayashida W, Hess OM, Douglas PS, Gordon S, Keighley CS, Benedict C, Krayenbuehl HP. Intracardiac angiotensin-converting enzyme inhibition improves diastolic function in patients with left ventricular hypertrophy due to aortic stenosis. Circulation 1994; 90: 2761-2771.

17 Chen CH, Nakayama M, Talbot M, Nevo E, Fetics B, Gerstenblith G, Becker LC, Kass DA. Verapamil acutely reduces ventricular-vascular stiffening and improves aerobic exercise performance in elderly individuals. J Am Coll Cardiol 1999; 33: 1602-1609.

18 Brutsaert DJ, Sys SU, Gillebert TC. Diastolic failure; pathophysiology and therapeutic implications. J Am Coll Cardiol 1992; 22: 318-325.

19 Yamagishi S, Inagaki Y, Nakamura K, Imaizumi T. Azelnidipine, a newly developed longacting calcium antagonist, inhibits tumor necrosis factor-alpha-induced interleukin-8 expression in endothelial cells through its anti-oxidative properties. J Cardiovasc Pharmacol 2004; 43: 724-730.

20 Nishikawa N, Masuyama T, Yamamoto K, Sakata Y, Mano T, Miwa T, Sugawara M, Hori M. Long-term administration of amlodipine prevents decompensation to diastolic heart failure in hypertensive rats. J Am Coll Cardiol 2001; 38: 1539-1545.

21 Yusuf S, Pfeffer MA, Swedberg K, Granger CB, Held P, McMurray JJ, Michelson EL, Olofsson B, Ostergren J. Effects of candesartan in patients with chronic heart failure and preserved left-ventricular ejection fraction: the CHARM-Preserved Trial. Lancet 2003; 362: 777-781.

22 Cleland JG, Tendera M, Adamus J, Freemantle N, Polonski L, Taylor J. The perindopril in elderly people with chronic heart failure (PEP-CHF) study. Eur Heart J 2006; 27: 2338-2345.

23 Schmieder RE, Matus $\mathrm{P}$, Klingbeil A. Reversal of left ventricular hypertrophy in essential hypertension. A meta-analysis of randomized double-blind studies. JAMA 1996; 275: 1507-1513.

24 Brilla CG, Funck RC, Rupp H. Lisinopril-mediated regression of myocardial fibrosis in patients with hypertensive heart disease. Circulation 2000; 102: 1388-1393.

25 Davis BR, Kostis JB, Simpson LM, Black HR, Cushman WC, Einhorn PT, Farber MA, Ford CE, Levy D, Massie BM, Nawaz S. Heart failure with preserved and reduced left ventricular ejection fraction in the antihypertensive and lipid-lowering treatment to prevent heart attack trial. Circulation 2008; 118: 2259-2267.

\section{APPENDIX}

\section{CALVLOC trial investigators}

Atsuhito Otuska, MD (Ibaraki Iseikai Hospital, Ibaraki, Japan); Kou Fujisawa, MD (Iwasa Dai-ichi Hospital, Gifu, Japan); Yorihiko Higashimo, MD (Higashi-Takarazuka Sato Hospital, Takarazuka, Japan); Motoo Date, MD (Sakurabashi Watanabe Hospital, Osaka, Japan); Kei Tawarahara, MD (Hamamatsu Red-cross Hospital, Hamamatsu, Japan); Mikio Mukai, MD (Kinki Chuo Hospital, Itami, Japan); Masanori Shinoda, MD (Kouseiren Kamo General Hospital, Toyota, Japan); Taro Minagawa, MD (Minagawa Clinic, Gifu, Japan); and Naoki Goto, MD (Goto Clinic, Gifu, Japan). 